\title{
X-Rays and $\gamma$-Rays from SN 1987A
}

\author{
By S. K U M A G A I \\ University of Tokyo, Bunkyo-ku, Tokyo 113, Japan
}

Theoretical light curves and spectra of X-rays and $\gamma$-rays from SN 1987A are calculated by the Monte Carlo method, based on a model built up from the early observations of neutrinos and optical light. Comparison of the predicted radiation with observational results obtained later confirms the radiation mechanism of supernovae: $\gamma$-rays are emitted in the decays of radioactive ${ }^{56} \mathrm{Co}$ and $\mathrm{X}$-rays are generated by the Compton degradation of these $\gamma$-rays. It also suggests that large scale mixing occurred and clumpy structure was formed inside the ejecta. These findings lead us to construct the model with a new distribution of elements, which is determined through comparisons of observations of X-rays and $\gamma$-rays with numerical simulations based on the assumed distribution. Using this model, the subsequent $\mathrm{X}$-ray and $\gamma$-ray emission is predicted: the light curves of $\mathrm{X}$-rays and $\gamma$-rays as well as their spectral evolution are in very good agreement with that expected from the radioactive decays of ${ }^{56} \mathrm{Co}$ and ${ }^{57} \mathrm{Co}$. The mass of newly synthesized ${ }^{44} \mathrm{Ti}$ and the emission from the neutron star will be determined by future satellite and balloon-borne observations.

\section{Introduction}

SN 1987A has given us an invaluable chance to examine supernova theory, which has predicted the emergence of $\mathrm{X}$-ray and $\gamma$-ray radiation from supernovae. Several possible mechanisms for the X-ray and $\gamma$-ray emission have been discussed, such as collision of the ejecta with circumstellar matter, nonthermal radiation from a pulsar, and Compton degradation of the line $\gamma$-rays emitted by radioactive nuclei. The observed light curve clarified the energy source of the radiation. It started to show the exponential decline $\sim 120$ days after the explosion with an e-folding time of 111.3 days (Catchpole et al. 1987; Hamuy et al. 1988). Since this time agrees with the lifetime for ${ }^{56}$ Co to decay into ${ }^{56} \mathrm{Fe}$, we infer that the dominant energy source of the optical radiation at this stage must be the decay of ${ }^{56} \mathrm{Co}$. The ${ }^{56} \mathrm{Co}$ is believed to come from the $8.8 \mathrm{~d}$ decay of ${ }^{56} \mathrm{Ni}$, which is produced by the explosive nucleosynthesis and powers the light curve in the earlier stage. The actual mechanism of the X-ray and $\gamma$-ray emission from SN 1987A was found to be Compton degradation of line $\gamma$-rays emitted at the decays of ${ }^{56} \mathrm{Co}$. During the first few hundred days after the explosion, the ejecta absorbs most of the energy released by the decay of ${ }^{56} \mathrm{Co}$ and emits it again in ultraviolet, optical and infrared bands (see McCray 1993). However, as the optical depth of the Compton scattering becomes small due to the expansion of the ejecta, (McCray et al. 1987) X-rays from SN $1987 \mathrm{~A}$ were expected to become observable by Ginga and Kvant (M. Itoh et al. 1987). The line $\gamma$-rays were also predicted to be detectable by the satellite MIR and Solar Maximum Mission (Gehrels et al. 1987; Chan and Lingenfelter 1987). These results mandate a more detailed prediction of the light curves of X-rays and $\gamma$-rays based on a more realistic model of SN 1987A.

\section{Hydrodynamical Model of the Ejecta}

To predict the radiation from SN 1987A, a model of SN 1987A proposed by Shigeyama et al. (1988) and Nomoto et al. (1987a) is employed. The progenitor was a star with $\sim 20 M_{\odot}$ on the main sequence and developed a helium core of $\sim 6 M_{\odot}$ as inferred from the luminosity of SK-69 202 (Woosley et al. 1988b). 


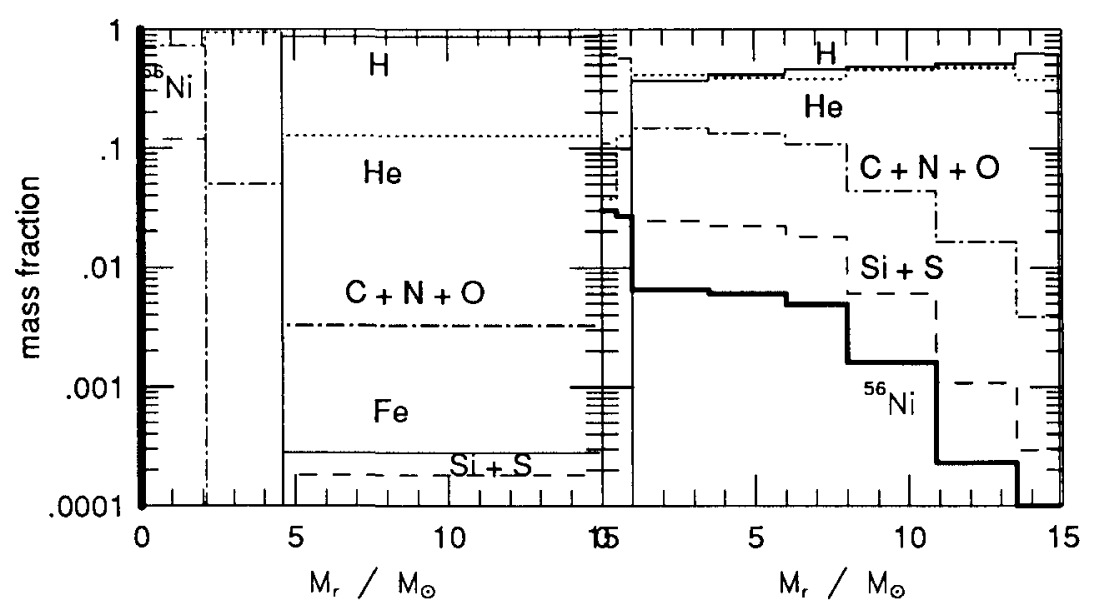

FIgURE 1. The distribution of chemical abundance of model 14E1 before mixing (left; Hashimoto et al 1989) and after mixing (right).

A neutron star with mass $1.4 M_{\odot}$ was formed at the explosion, which is inferred from the observed neutrino flux. the chemical composition of the ejecta after the explosive nucleosynthesis is shown in Fig. 1 (left). The ejecta consist of a heavy element core of $2.4 M_{\odot}$ (composed of $0.073 M_{\odot}{ }^{56} \mathrm{Ni}, 0.26 M_{\odot}$ Ar-S-Si and $2.07 M_{\odot} \mathrm{Mg}-\mathrm{Ne}-\mathrm{O}-\mathrm{C}$ ), a $2.2 M_{\odot}$ He-rich layer ( $4 \%$ carbon and $1 \%$ oxygen in mass fraction) and a $\mathrm{H}$-rich envelope of mass $10.3 M_{\odot}$ (including heavy elements of $1 / 4$ times the solar abundance). Using the model of the ejecta 14E1 (Nomoto et al. 1987a; Shigeyama et al. 1988), Monte Carlo simulations are performed to follow the trails of photons inside the ejecta. In evaluating the flux at Earth, we assume the distance to the supernova to be $55 \mathrm{kpc}$.

\section{X-Ray and $\gamma$-Ray Light Curves}

\subsection{The effects of mixing}

Comparison of the resulting X-ray light curve (dotted curve in Fig. 2) with the early Ginga observations (crosses; Dotani et al. 1987), suggests large scale mixing in the ejecta (M. Itoh et al. 1988; Kumagai et al. 1988a, 1992; Nomoto et al. 1991a, 1991b). Monte Carlo simulations are conducted assuming the region of mixing, and the results show that the model with mixing at $M_{r}<13.5 M_{\odot}$ most consistently reproduces the light curve (dashed curve in Fig. 2) with the Ginga observation till $300^{\mathrm{d}}$ after the explosion. The resulting chemical composition is shown in Fig. 1 (right). After mixing, ${ }^{56} \mathrm{Ni}$ is mixed up to $M_{r}=13.5 M_{\odot}$ where the expansion velocity of the material amounts to $4200 \mathrm{~km} \mathrm{~s}^{-1}$. The column depth to this layer is $2.4 \mathrm{~g} \mathrm{~cm}^{-2}$ at $t=200^{\mathrm{d}}$. At early times, the emergent $\mathrm{X}$-rays originate from ${ }^{56} \mathrm{Co}$ at the outermost layers. Later, the $\gamma$-rays and $\mathrm{X}$-rays from ${ }^{56} \mathrm{Co}$ in the deeper layer contribute. Though the mass fraction of original ${ }^{56} \mathrm{Co}$ is much larger in the core than in the envelope, the emergent flux does not increase so steeply because most of the ${ }^{56} \mathrm{Co}$ has already decayed and the absorption by the core material is larger. Thus, the X-ray light curve shows a relatively broad peak. Accordingly, the dashed curve is in good agreement with the Ginga observations up to $\sim 300^{\mathrm{d}}$.

Fig. 3 shows the calculated line $\gamma$-ray light curves based on the above chemical distribution. They are all consistent with $S M M$ and balloon borne observations. The early emergence of $\gamma$-rays in the model is due to the mixing of ${ }^{56} \mathrm{Co}$ out to $M_{r} \sim 13.5 M_{\odot}$. 


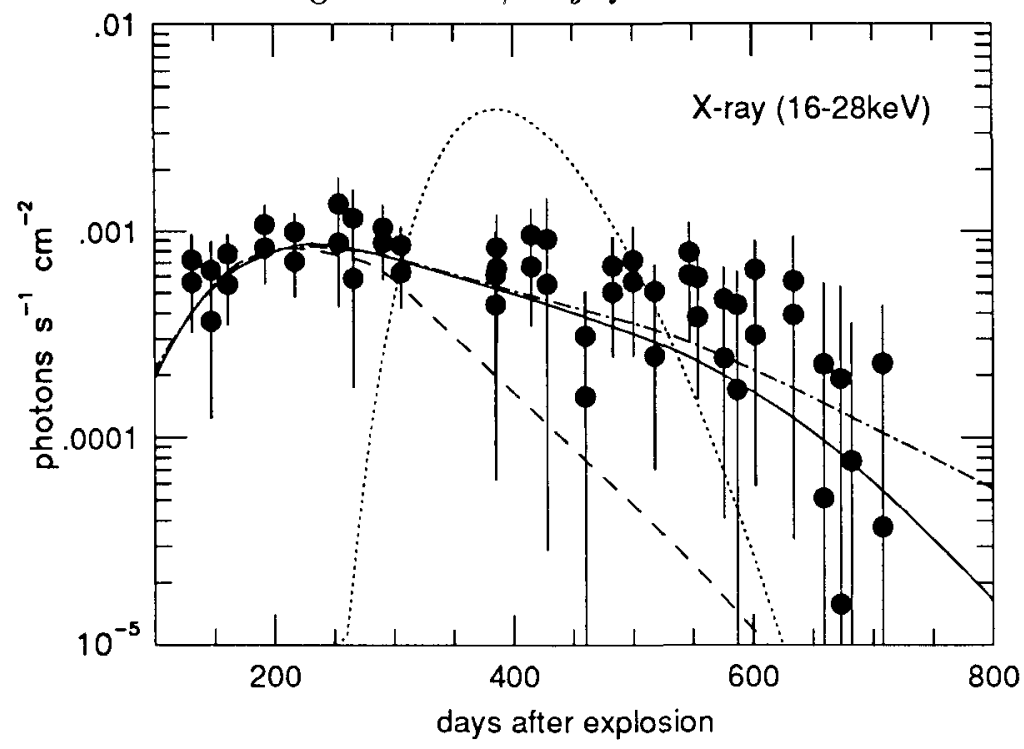

Figure 2. X-ray light curves calculated by Monte Carlo simulation based on the model 14E1 (Nomoto et al. 1987a: Shigeyama et al. 1988) The dotted curve is in the case without mixing and the dashed curve corresponds to spherical mixing at $M_{r}<13.5 M_{\odot}$. The solid curve is for the model with the reduction of the photo-electric opacity by a factor 10 at $M_{r}<8 M_{\odot}$ in addition to mixing and the dash-dotted curve is obtained by taking account of the effect of ${ }^{57} \mathrm{Co}$, comparing the Ginga observation (Inoue 1991).

The flux ratio between $847 \mathrm{keV}$ and $1238 \mathrm{keV}$ line $\gamma$-rays is close to unity at early stages because of the smaller cross section for $1238 \mathrm{keV}$ than for $847 \mathrm{keV}$. It approaches the experimental value of 0.68 as the column depth decreases.

The predicted light curves of line $\gamma$-rays from the decays of ${ }^{57} \mathrm{Co}$ and ${ }^{44} \mathrm{Ti}$ are shown in Fig. 4 (Kumagai et al. 1992). Recently, the Compton Gamma Ray Observatory detected the $122 \mathrm{keV}$ line originating from ${ }^{57} \mathrm{Co}$ (Kurfess et al. 1992). These observations imply that the isotope ratio of ${ }^{57} \mathrm{Ni}$ and ${ }^{56} \mathrm{Ni}$ synthesized at the explosion $1.5 \pm 0.5$ times the solar ratio, which is consistent with the velue 1.7 from nucleosynthesis theory. Future observations of these lines should confirm the amount of radioactive nuclei and, hence, the nucleosynthesis theory.

\subsection{The effects of clumps}

At later times, $t>300^{\mathrm{d}}$, the $16-28 \mathrm{keV} \mathrm{X-ray} \mathrm{flux} \mathrm{observed} \mathrm{by} \mathrm{Ginga} \mathrm{(Inoue} \mathrm{1991)}$ declines very slowly, while the calculated X-ray flux (the dashed curve in Fig. 2) decreases significantly faster than the observation. To be more realistic, we should take account of the effect of clumps on the X-ray absorption in the core, though the above calculation assumes homogeneous and spherically symmetric mixing, which maximizes the absorption.

If the heavy elements are localized in particular clumps, a large fraction of the X-rays could be transported through the hydrogen and helium-rich regions without suffering much photoelectric absorption; this would effectively reduce the opacity. A calculation assuming the photoelectric opacity to be reduced by a factor 10 in $M_{r}<8 M_{\odot}$ gives the $\mathrm{X}$-ray light curve most consistent with the Ginga observation (solid curve in Fig. 2). The most probable mechanism to mix the supernova ejecta is the Rayleigh-Taylor instability. Numerical simulations of such mixing have been carried out by Arnett et al. (1989b), Hachisu et al. (1989). For example, Hachisu et al. (1989) shows that the materials of Si 


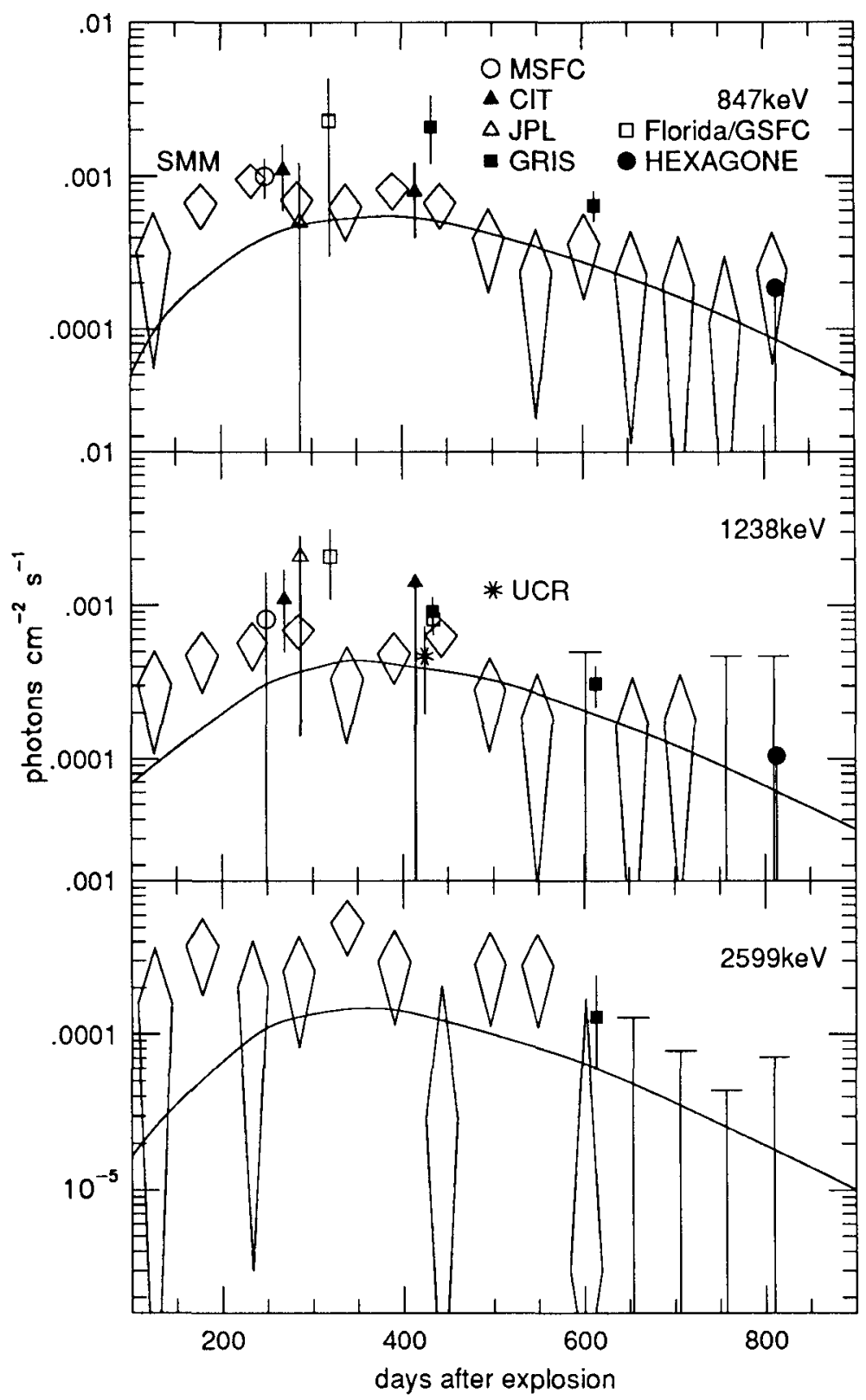

Figure 3. The calculated and observed line $\gamma$-ray light curves of $847 \mathrm{keV}$ (upper), $1238 \mathrm{keV}$ (middle), and $2599 \mathrm{keV}$ (lower) of model 14E1 comparing with the observation of SMM (diamonds: Gehrels et al. 1987) and balloons (crosses: Sandie et al. 1988; Cook et al. 1988; Mahoney et al. 1988; Rester et al. 1989).

and O-rich layer are mixed up to $M_{r}=8 M_{\odot}$, where the expansion velocity is $\sim 2200 \mathrm{~km}$ $\mathrm{s}^{-1}$, and that hydrogen is mixed down to $M_{r}=1 M_{\odot}$ at the same time. These results can explain the occurrence of mixing in the ejecta, but cannot completely reproduce the extent of ${ }^{56} \mathrm{Co}$ mixing to outer layers presented by the X-ray, $\gamma$-ray, and infrared observations.

The dash-dotted curve in Fig. 2 adds a contribution from the decay of ${ }^{57} \mathrm{Co}$ to the 



Figure 4. (top) The calculated line $\gamma$-ray light curves of $122 \mathrm{keV}$ (solid curve) from ${ }^{57}$ Co decay is compared with the upper limits obtained from the balloon experiments (GKM: Gunji et al. 1992; HEXAGONE: Chapuis et al. 1993) and the flux translated from the OSSE experiments (Kurfess et al. 1992). The light curves of $136 \mathrm{keV}$ (dash-dotted curve), and $14 \mathrm{keV}$ (dashed curve) are also shown. (bottom) The calculated line $\gamma$-ray light curves of $1154 \mathrm{keV}$ (solid curve), $511 \mathrm{keV}$ (dash-dotted curve), $67.9 \mathrm{keV}$ and $78.4 \mathrm{keV}$ (dotted curves) from ${ }^{44} \mathrm{Ti}$, compared with the upper limit obtained with HEXAGONE (Chapuis et al. 1993).

solid curve, which corresponds to ${ }^{56} \mathrm{Co}$ only. This additional X-ray flux exceeds that from the ${ }^{56}$ Co decay at $t>600^{\mathrm{d}}$ and slows down the decline of the $\mathrm{X}$-ray light curve. However, the effect is not sufficiently large and a reduction of the photo-electric opacity is still necessary to account for the observations for $t>300^{\mathrm{d}}$.

Calculated X-ray light curves of higher energy bands adding the effect of ${ }^{57} \mathrm{Co}$, based on the mixing model obtained above are shown In Fig. 5. They also agree very well with the MIR observations (Sunyaev et al. 1988). 


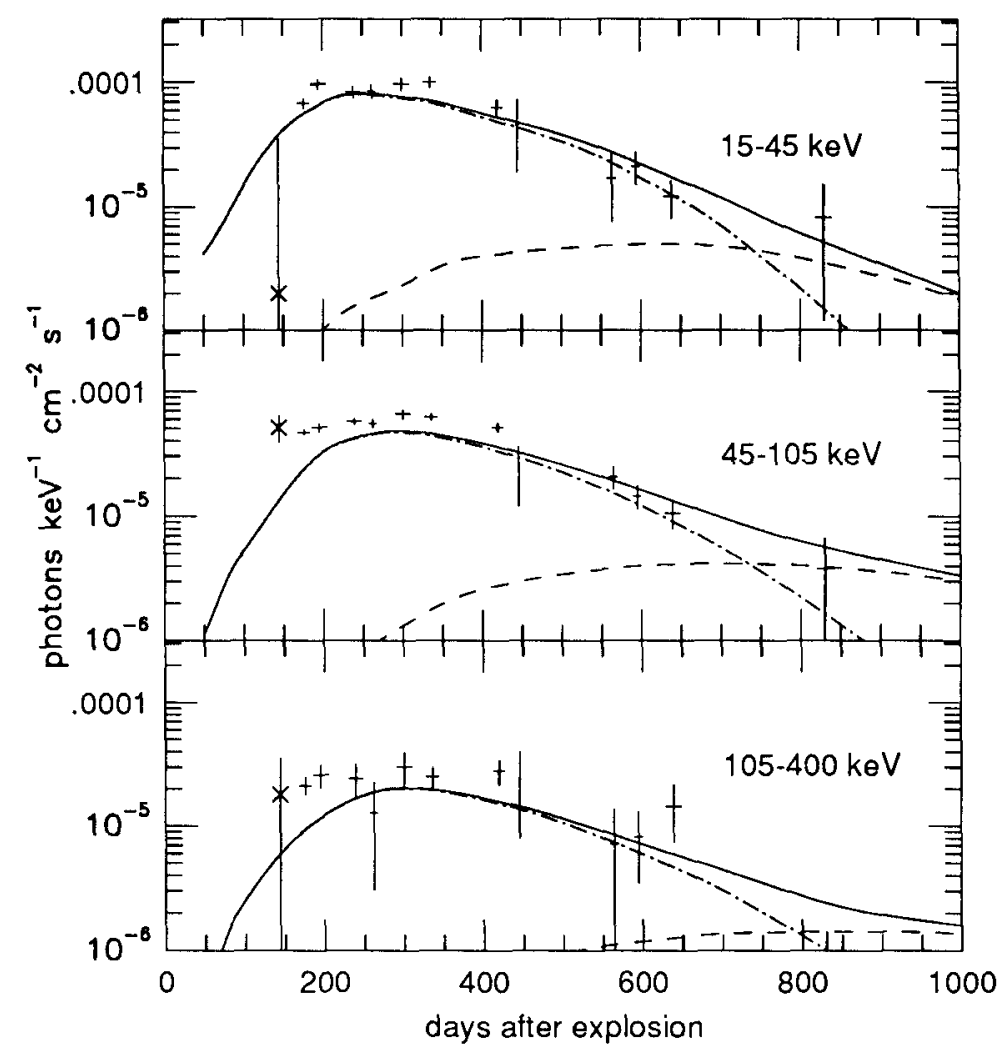

Figure 5. Calculated and observed light curves of $15-45 \mathrm{keV}$ (upper), $45-105 \mathrm{keV}$ (middle) and $105-400 \mathrm{keV}$ (lower). Dash-dotted lines and dashed lines are the contributions of ${ }^{56} \mathrm{Co}$ and ${ }^{57} \mathrm{Co}$, respectively, and the solid lines are their sum. The MIR observations are also shown for comparison (Sunyaev et al. 1988, 1989).

\section{X-Ray and $\gamma$-Ray Spectra}

It is also important to compare theoretical calculations of the hard X-ray and $\gamma$-ray spectra from the decays of ${ }^{56} \mathrm{Co}$ and ${ }^{57} \mathrm{Co}$ with observations. Fig. 6 shows this comparison. The calculated spectra for $E \gtrsim 15 \mathrm{keV}$ are in good agreement with the observations, which implies that the down-scatterings of $\gamma$-rays and hard X-rays in SN 1987A are well modeled by our calculation. The theoretical spectrum does not appreciably change until $t \sim 400^{\mathrm{d}}$ and becomes harder as the ejecta expands and the number of Compton scattering decreases.

The dash-dotted curves in Fig. 6 show the emergent spectra due to the degraded line $\gamma$-rays from the ${ }^{57} \mathrm{Co}$ decay. Because ${ }^{57} \mathrm{Co}$ has a longer half life $\left(271^{\mathrm{d}}\right)$ than ${ }^{56} \mathrm{Co}\left(78^{\mathrm{d}}\right)$, the X-rays below $122 \mathrm{keV}$ are dominated by the ${ }^{57} \mathrm{Co}$ component for $t>600^{\mathrm{d}}$. This fact is evident in the dash-dotted and solid curves in Fig. 2. It is interesting to compare the predicted hard X-ray spectrum at $t=600^{\mathrm{d}}$ with HEXE observations (Fig. 6; Sunyaev et al. 1989). Without a contribution from ${ }^{57} \mathrm{Co}$ decay, the theoretical flux below $100 \mathrm{keV}$ is about a factor of 2 smaller than the observed flux. With the adopted abundance of ${ }^{57} \mathrm{Ni}$, the contributions from the decays of ${ }^{56} \mathrm{Co}$ and ${ }^{57} \mathrm{Co}$ are comparable at $t \sim 600^{\mathrm{d}}$; this choice gives excellent agreement between the predicted and the observed flux, as seen in Fig. 6. This suggests that the abundance ratio of ${ }^{57} \mathrm{Ni} /{ }^{56} \mathrm{Ni}$ may be about twice as large as the solar ratio as calculated by Hashimoto et al. (1989) (see also Sunyaev et al. 1989).

On the other hand, the Compton-degraded $\gamma$-rays cannot account for the X-rays below 


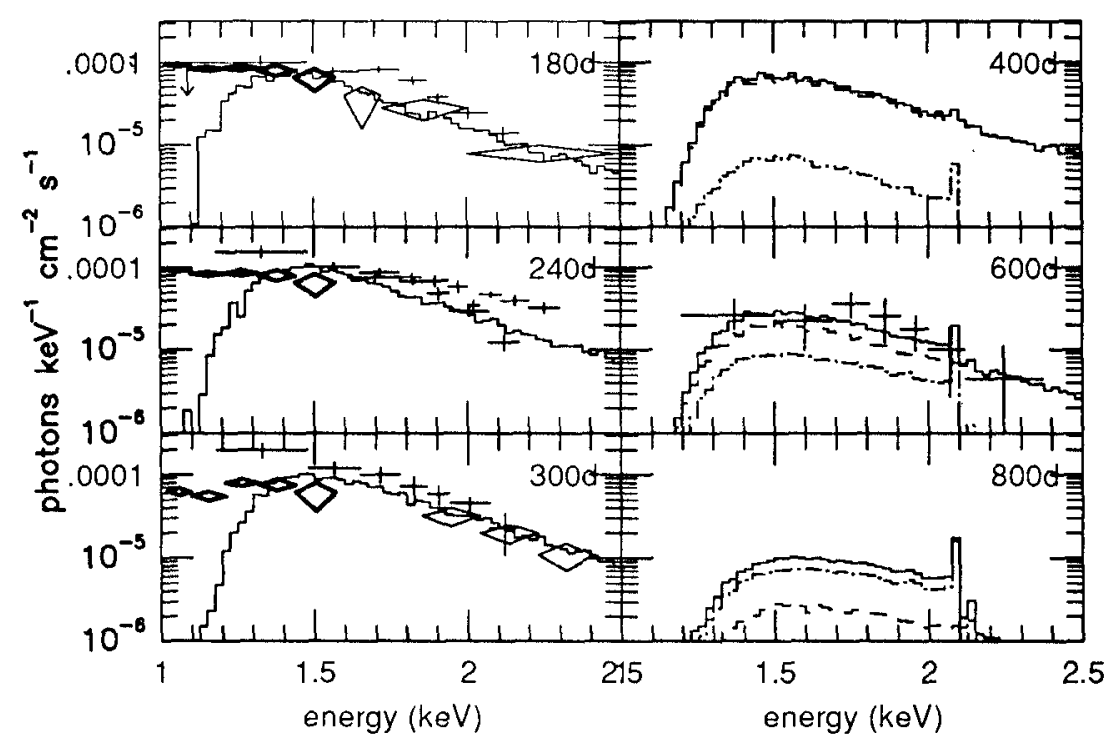

Figure 6. Calculated hard X-ray and $\gamma$-ray spectra due to the decays of ${ }^{56} \mathrm{Co}$ (solid curve) and ${ }^{57} \mathrm{Co}$ (dash-dotted curve) for model $14 \mathrm{E} 1$ with the photoelectric opacity reduced by a factor 10 for $M_{r}<8 M_{\odot}$. The thin diamonds show the spectra observed by Ginga (Inoue 1991); the thick diamonds and the thick crosses are observations with Pulsar X-1 and HEXE on Kvant (Sunyaev et al. 1987, 1989); and the thin crosses are balloon-borne observations (Wilson et al. 1988). For the figures of $t=400^{\mathrm{d}}, 600^{\mathrm{d}}$, and $800^{\mathrm{d}}$, the ${ }^{56} \mathrm{Co}$ and ${ }^{57} \mathrm{Co}$ components are shown by the dashed and dash-dotted curves, respectively, and the solid curve is their sum.

$16 \mathrm{keV}$ observed by Ginga, which show the time variations of the intensity and spectrum. Instead, thermal emission from the ejecta heated by collision with preexisting circumstellar matter reproduces them very well (see H. Itoh et al. 1987; Masai et al. 1987, 1988).

\section{Conclusions}

Through the comparison of the theoretical light curves and spectra of X-rays and $\gamma$ rays from SN 1987A with observational results obtained later, we reach the following conclusions: (1) The high energy radiation mechanism of supernovae is confirmed: $\gamma$ rays are emitted at the decays of radioactive ${ }^{56} \mathrm{Co}$ and $\mathrm{X}$-rays are generated as Compton degraded $\gamma$-rays. (2) A new model of supernova ejecta is constructed, in which a large scale mixing occurs and clumpy structure is formed. This model reproduces very well the optical and infrared observation. (3) The theoretical UV-IR light curve based on the above model is predicted to decay faster than exponential decline of ${ }^{56} \mathrm{Co}$ at $\sim 300$ days after the explosion, because a larger fraction of X-rays and $\gamma$-rays escape from the ejecta in this stage. This was confirmed later by the observations at SAAO, ESO, and CTIO.

With the new model described above, future $\mathrm{X}$-rays and $\gamma$-rays originating from other radioactive nuclei with longer half lives, such as ${ }^{57} \mathrm{Co}$ and ${ }^{44} \mathrm{Ti}$, and a buried neutron star are predicted. The $122 \mathrm{keV}$ line $\gamma$-ray from ${ }^{57} \mathrm{Co}$ has been detected by the Compton Gamma Ray Observatory and its intensity is consistent with the prediction. The intensities of line $\gamma$-rays from ${ }^{44} \mathrm{Ti}$ should become almost constant for post-explosion times greater than 5 years and should be observable with future satellites. If a pulsar 
with a luminosity of $10^{37} \mathrm{erg} \mathrm{s}^{-1}$ exists, the X-ray flux will become intense enough to be observable with $A S C A$ in $\sim 1995$ and with $A S T R O-E$ in $\sim 2000$.

\section{Acknowledgements}

It is a great pleasure to thank Professors K. Nomoto, J. Nishimura, Drs. M. Itoh and $\mathrm{T}$. Shigeyama for continuous discussion and valuable comments. I am also deeply grateful to Prof. McCray for offering me an opportunity to attend the meeting.

\section{REFERENCES}

Arnett, W. D. 1988, ApJ, 331, 337

Arnett, W. D. \& Fu, A. 1989a, ApJ, 340, 396

Arnett, W. D., Fryxel, B. A. \& Müller, E. 1989b, ApJ, 341, 163

Bouchet, P., Danziger, I. J. \& Lucy, L. 1991a, In SN 1987A and other Supernovae, ed. I. J. Danziger (ESO; Garching), p. 281.

Bouchet, P., Danziger, I. J. \& Lucy, L. 1991b, AJ, 102, 1135

Burrows, A. \& Lattimer, J. M. 1987, ApJ, 318, L63

Burrows, A. \& The, L.-S. 1990, AJ, 100, 1575

Catchpole, R. M. et al. 1987, MNRAS, 229, 15

Catchpole, R. M. et al. 1988, MNRAS, 231, 75

Catchpole, R. M. et al. 1989, MNRAS, 237, 55

Chan, K.W. \& Lingenfelter, R.E. 1987, ApJ, 318, L51

Chevalier, R. A. \& Fransson, C. 1987, Nature, 328, 44

Cook, W. R. et al. 1988, ApJ, 334, L87

Danziger, I. J. et al. 1989, In Big Bang, Active Galactic Nuclei and Supernovae, ed. S. Hayakawa and K. Sato (Universal Academy Press; Tokyo), p. 249.

Dotani, T. et al. 1987, Nature, 330, 230

Ensman, L. \& Woosley, S. E. 1988, ApJ, 333, 754

Filippenko, A. V., Porter, A. C. \& Sargent, W. L. W. 1990, AJ, 100, 1575

Gehrels, N., MacCallum, C. J. \& Leventhal, M. 1987, ApJ, 320, L19

Hamuy, M., Suntzeff, N. B., Gonzalez, R. \& Martin, G. 1988, AJ, 95, 63

Hashimoto, M., Nomoto, K. \& Shigeyama, T. 1989, A\&A, 210, L5

Inoue, H. et al. 1991, PASJ, 43, 213.

Itoh, H., Hayakawa, S., Masai, K. \& Nomoto, K. 1987, PASJ, 39, 529

Itoh, M. et al. 1987, Nature, 330, 233

Itoh, M. et al. 1988, In Atmospheric Diagnostics of Stellar Evolution, ed. K. Nomoto (Springer; Berlin), Lecture Notes in Physics, 305, pp. 446

Kirshner, R. et al. 1987, ApJ, 320, 602

Kumagai, S. et al. 1988a, A\&A, 197, L7

Kumagai, S. et al. 1988b, In Supernovae 1987A in the Large Magellanic Cloud, ed. M. Kafatos and A. Michalitsianos (Cambridge University Press; Cambridge), p. 414.

Kumagai, S. et al. 1989, ApJ, 345, 412

Kumagai, S., Shigeyama, T. \& Nomoto, K. 1991a, In SN 1987A and other Supernovae, ed. I.J. Danziger, (ESO; Garching), p. 203

Kumagai, S. et al. 1991b, A\&A, 243, L13

Kumagai, S. \& Nomoto, K. 1991c, In Frontiers of X-ray Astronomy, ed. Y. Tanaka (Universal Academy Press; Tokyo), p. 375 
Kumagai, S. 1992, Ph.D. thesis, University of Tokyo. In Frontiers of X-ray Astronomy, ed. Y. Tanaka (Universal Academy Press; Tokyo), p. 375

Kurfess, J. D. et al. 1992, ApJ, 399, L137.

Leibundgut, B. et al. 1991, ApJ, 371, L23

Mahoney, W. A. et al. 1988, ApJ, 334, L81

Makino, F. et al. 1987, ApJ, 25, 223

Masai, K. et al. 1988, Nature, 335, 804

McCray, R., Shull, J. M. \& Sutherland, P. 1987, ApJ, 317, L73

McCray, R. 1993, ARA\&A, 31, 175

Nagasawa, M. et al. 1988, PASJ, 40, 691

Nomoto, K. et al. 1987, In SN 1987A, ed. I. J. Danziger, (ESO; Garching), p. 325.

Nomoto, K. et al. 1991a, in Supernovae, ed. S.E. Woosley (Springer), p. 176

Nomoto, K. et al. 1991b, In Supernovae and Stellar Evolution, eds. A. Ray and T. Velusamy (World Scientific; Singapore), p. 116

Rester, A. C. et al. 1989, ApJ, 342, L71

Sandie, W. G. et al. 1988, ApJ, 334, L91

Shigeyama, T. et al. 1987, Nature, 328, 320

Shigeyama, T., Nomoto, K. \& Hashimoto, M. 1988, A\&A, 196, 141

Suntzeff, N.B. et al. 1991, AJ, 102, 1118

Sunyaev, R. A. et al. 1987, Nature, 330, 227

Sunyaev, R. A. et al. 1988, Soviet Ast. Lett., 14, 247

Sunyaev, R. A. et al. 1989, Soviet Ast. Lett., 15, 291

Wheeler, J. C. \& Harkness, R. 1990, Rep. Prog. Phys., 53, 1467

Whitelock, P. A. et al. 1988) MNRAS, 234, 5

Wilson, R. B. et al. 1988, In Nuclear Spectroscopy of Astrophysical Sources, ed. N. Gehrels and G. Share (AIP; New York) p. 6655

Woosley, S. E. \& Weaver, T.A. 1986, ARA\&A, 24, 205

Woosley, S. E., Pinto, P. A., Martin, P. G. \& Weaver, T. A. 1987, ApJ, 318, 664

Woosley, S. E., Pinto, P. \& Ensman, L. 1988, ApJ, 324, 466

Woosley, S. E. 1988, ApJ, 330, 218

Xu, Y., Sutherland, P., McCray, R. \& Ross, R. R. 1988, ApJ, 327, 197 